\section{TRANSACCIONALISMO, INTERSECCIONALIDAD FEMINISTA Y MÉTODO NARRATIVO: APORTES PARA LA INVESTIGACIÓN EN TERAPIA OCUPACIONAL Y CIENCIA OCUPACIONAL*}

Transacionalismo, Interseccionalidade Feminista e Método Narrativo: aportes para a pesquisa em Terapia Ocupacional e Ciência Ocupacional

Transactionalism, Feminism Intersectionality and Narrative Method: contributions for research in Occupational Therapy and Occupational Science

\section{Resumen}

Introducción. Las investigaciones en terapia y ciencia ocupacional son cada vez más diversificadas y complejas. Actualmente, se busca superar la perspectiva única e individual, focalizada principalmente en "la persona", pasando a considerar el contexto como parte de un complejo entramado social y colectivo. Óbjetivo. El presente escrito busca presentar dos teorías que son útiles al ámbito investigativo de la terapia ocupacional y la ciencia ocupacional, una más consolidada en la disciplina - el Transaccionalismo y otra que podría generar interesantes discusiones en distintas perspectivas e investigaciones, la Interseccionalidad feminista. Además, junto a lo anterior, presenta las narrativas como una metodología que permita la integración de ambas propuestas. Método. Se realiza una revisión teórica desde un análisis crítico, basado en investigaciones teórico-prácticas. Resultados y discusiones. Buscamos analizar como la integración entre las perspectivas transaccionalistas, interseccionales y metodologías cualitativas, en especial los relatos de vida y narrativas, pueden ofrecer aportes teórico-metodológicos para la investigación en terapia ocupacional y en ciencia ocupacional, de forma de producir saberes y acciones críticas, coherentes y complejas, con foco en el estudio de las ocupaciones, necesarias para nuestra actualidad global. Conclusiones. Se concluye que las investigaciones que busquen caracterizar las ocupaciones de las personas desde perspectivas más complejas, podrían integrar algunos de estos aspectos teóricos, pues facilitan la comprensión y problematización de las diferentes ocupaciones desde perspectivas colectivas, sociales, históricas y contextualizadas. Palabras claves: Construcción de conocimiento; Ciencia; Historia de vida; Interseccionalidad, Feminismo, Ocupación; Perspectiva Crítica; Transaccionalismo.

\section{Resumo}

Introdução. As investigações em terapia ocupacional e em ciência ocupacional são cada vez mais diversificadas e complexas. Atualmente se busca superar a perspectiva única e individual focalizada, principalmente 'na pessoa', passando a considerar o contexto como parte de uma complexa trama social e coletiva. Objetivo. O presente artigo busca apresentar duas teorias que são uteis no âmbito investigativo da terapia ocupacional e da ciência ocupacional, uma mais consolidada na TO - o Transacionalismo e outra que pode promover interessantes discussões em diferentes perspectivas e pesquisas, a Interseccionalidade Feminista. Além disso, nesta direção, apresenta as narrativas como uma metodologia que permite a integração de ambas perspectivas. Método. Foi realizada uma revisão teórica a partir de análises críticas e baseadas em pesquisas teórico-práticas. $R e$ sultados e Discussões. Buscamos analisar como a integração entre as perspectivas transaccionalistas, interseccionais e metodologias qualitativas, em especial os relatos de vida e narrativas, podem oferecer aportes teórico-metodológicos para a investigação em terapia ocupacional e em ciência ocupacional, de forma de produzir saberes e ações críticas, coerentes e complexas, com foco nos estudos das ocupações, necessários para nossa atualidade global. Conclusões. Conclui-se que as investigações que buscam caracterizar as ocupações das pessoas desde perspectivas mais complexas, poderiam integrar as perspectivas teóricas apresentadas, pois facilitam a compreensão e problematização das diferentes ocupações desde perspectivas coletivas, sociais, históricas e contextualizadas.

Palavras chaves: Construção de conhecimento; Ciência; Interseccionalidade, Feminismo; Ocupação; Perspectiva crítica; História de vida, Transacionalismo.

\section{Abstract}

Introduction. Research in occupational therapy and occupational science are increasingly diversified and complex, part of the search to overcome the unique and individual perspective with a central focus only on "the person", and consider the context as part of a complex structure social and collective. Objective. Or present paper seeks to present theories that are useful not research field of occupational therapy and occupational science, one more consolidated, the Transactionalism and another one that can promote interesting discussions in different perspectives and researches, Feminism Intersectionality. In addition, this paper presents the narratives as a methodology that allows the integration of both perspectives. Method. The paper is based on critical analyzes and theoretical studies, based on theoretical-practical research. Results and Discussions. The paper seek to analyse how the integration between the Transactionalism, Intersectionality and qualitative methodologies, especially the life stories and narratives, can offer theoreticalmethodological contributions for research in occupational therapy and occupational science, in order to produce knowledge and critical actions, coherent and complex, focusing on the study of the occupations, necessary for our global news. Conclusions. It is concluded that the researches that seek to characterize the occupations of people from more complex perspectives, could integrate some of these theoretical aspects, because they facilitate the understanding and problematization of the different occupations from collective, social, historical and contextualized perspectives.

Keywords: Intersectionality; feminism; knowledge construction; science; occupation; critical perspective, life history; Transactionalism. 


\section{INTRODUCCIÓN}

Las investigaciones en terapia ocupacional (TO) y ciencia ocupacional (CO) están cada vez más diversificadas y complejas. Atrás ha quedado la perspectiva única e individual de ambas disciplinas, donde el foco central se entendía únicamente como "la persona", pasando a considerar a esa persona en un contexto y como parte de un complejo entramado social y colectivo. En este camino, se ha descrito cómo el Paradigma Social de la Ocupación ${ }^{1,2}$ ha generado perspectivas más entrelazadas y coherentes para la comprensión del objeto de estudio e intervención de la terapia ocupacional y de la ciencia ocupacional, en la actualidad.

Desde esta mirada, se hace necesaria la búsqueda constante de nuevas teorías y perspectivas metodológicas que aporten al desarrollo disciplinar. Es así como, el transaccionalismo, epistemología muy en boga, especialmente en la ciencia ocupacional, ha tomado fuerte relevancia en los últimos años.

Diferentes publicaciones ${ }^{3-6}$ han tomado al transaccionalismo como un elemento importante para el análisis de las ocupaciones de las personas, lo que ha dejado en evidencia su gran contribución disciplinar.

Por otro lado, esta perspectiva también puede ser complementada desde miradas críticas que permitan identificar de forma más profunda los entramados que operan en las ocupaciones que las personas desempeñan día a día. En este camino, la interseccionalidad feminista ${ }^{7-10}$ podría resultar una importante contribución.

Además de lo anterior, es importante la combinación de una metodología que permitiría la integración de ambas miradas, como la narrativa ${ }^{11,12}$. Debido a que, como señalaremos más adelante, facilita una profundidad en la investigación que rompe con las dicotomías básicas de análisis más tradicionales.

En esta publicación describiremos estos tres aspectos, al transaccionalismo, la interseccionalidad feminista y a las narrativas, y - propondremos los aportes que estos constructos pueden otorgar a la terapia ocupacional y a la ciencia ocupacional.

\section{TRANSACCIONALISMO}

La forma tradicional de estudiar la ocupación, esto es desde perspectivas individuales ${ }^{13}$ o que solo consideran la forma, la función y el significado de las ocupaciones ${ }^{14}$ nos resultaba problemática pues implica una dualidad dicotómica implícita entre la persona y su contexto. Esto se relaciona con que, desde los inicios de los estudios en la ciencia ocupacional, la ocupación se ha entendido de manera individualista y centrada en la persona, como única componente generadora o "constructora" de ocupaciones ${ }^{15,16}$. 
Dickie, Cutchin y Humphry ${ }^{15}$ han analizado cómo la ocupación ha sido estudiada, desde definiciones como las de Wilcok, Yerxa, Christiansen, Clark, Nelson, entre otros, como un elemento individual, esto es propio de "la persona", donde es ella quien tiene la necesidad y el potencial de desempeñarla en su ambiente ${ }^{16}$. Estos/as autores/as refieren además, que estas definiciones posicionan la ocupación en la intersección de la persona y su contexto, pero es determinado por ella misma: "el estudio de la ocupación requiere el estudio de la persona como autor/a de su trabajo, descanso, juego, ocio y autocuidado" (p. 10) ${ }^{16}$

Concordamos con Dickie, Cutchin y Humphry ${ }^{15}$, que para analizar las experiencias ocupacionales de las personas es fundamental, no solo centrarse en las experiencias individuales, sino también en la relevancia, determinación e influencia de los diversos y diferentes contextos, además de la perspectiva histórico-social de la ocupación desempeña$\mathrm{da}^{17-19}$. De esta manera, proponemos que el estudio de las ocupaciones debe ser mucho más amplio que solamente la reducción del "desempeño de la persona en el ambiente", y que esta relación está influenciada o determinada por muchos otros factores (lo que podría relacionarse también con los principios de la perspectiva interseccional feminista que señalaremos más adelante).

Además, múltiples aspectos interrelacionados e involucrados pueden dar cuenta de un desempeño ocupacional particular que, puede reflejar segregación y discriminación ${ }^{20}$.

Para comprender mejor lo anterior, debemos entender que el transaccionalismo nace desde una perspectiva filosófica pragmatista. Dewey y Bentley comienzan a formar este concepto de "trans-acción" que pretende ir más allá de la "inter-acción", implicando al contexto, historia, expectativas e individuos de forma interrelacionada ${ }^{15}$.

Dewey define la posición transaccionalista desde los aspectos sociales, culturales, y los componentes morales de la acción, posicionándolos como elementos esenciales para comprender la experiencia humana en un mundo incierto y fluctuante ${ }^{4,21}$. Así, podemos analizar la coordinación funcional (acción) como transacción a través de la reestructuración dinámica y coordinada de la relación del sujeto con una situación particular, pues está en relación con las cosas, o sea, centrándose en las relaciones entre los objetos y no fuera de ellos, lo que se estudia en la transacción.

Por otro lado, Heidegger ${ }^{22}$ y Dewey ${ }^{23}$ proponen que los significados de las ocupaciones están situadas en contextos cotidianos de las personas y están conformadas por otros en el mundo ${ }^{16}$. Ambos filósofos refieren que estos significados se forman a partir: de la persona que realiza la ocupación y con quién la hace; de la situación en que las ocupaciones ocurren; y de cómo las ocupaciones son contextualizadas e integradas en el pasado, el presente y proyectadas hacia un futuro. Heidegger y Dewey brindan esta visión de ir más allá de la perspectiva individual, de ir hacia una perspectiva situada en el contexto de la persona y situada en el mundo ${ }^{16}$. 
3 INTERSECCIONALIDAD

La interseccionalidad feminista nace desde una crítica a los movimientos feministas blancos de mujeres de clase media en Estados Unidos que se centraban en las luchas de "ciertas mujeres" más visibilizadas que otras. ${ }^{2,2}$. En este contexto, durante los años 1980' las mujeres negras comienzan a manifestar que no se han identificado con las demandas de las luchas de "ciertas" feministas. Así, se dieron cuenta que, además de ser mujeres, existían otras condiciones dentro de su existencia que les otorgaban diferentes desigualdades, y que no estaban consideradas dentro de los movimientos en ese entonces ${ }^{7}$.

A partir de estos pensamientos e inspiradas en luchadoras negras desde los tiempos de la esclavitud, una de las primeras en acuñar este término fue Kimberlé Crenshaw, mujer, académica y afro estadounidense que comienza a visibilizar las problemáticas existentes dentro de nuevas dimensiones de empoderamiento negro feminino ${ }^{10}$.

De forma particular, una propuesta que permite evidenciar los diferentes sistemas y múltiples estructuras de opresión que influyen en la experiencia cotidiana de las personas, es la perspectiva interseccional de Patricia Hill Collins ${ }^{25}$. Ella propone el análisis de una matriz de dominación que sostiene un sistema de injusticias e inequidades basado en diferentes categorías e interrelaciones.

La propuesta de Hill Collins establece que "la interseccionalidad refiere formas particulares de opresiones que se cruzan, por ejemplo, intersecciones de raza y género, o de sexualidad y nación", y en ese sentido, "la opresión no puede reducirse a un tipo fundamental, (...) las opresiones trabajan juntas para producir la injusticia"25 (p. 18). Concretamente su propuesta radica en la conceptualización de los ejes de opresión que se entrelazan en el marco de una matriz de dominación, desde donde se constituyen las personas en distintas y cambiantes posiciones de poder.

Esta matriz de dominación explica la interacción e interdependencia entre líneas de opresión, y advierte la posibilidad de que las personas y grupos se encuentren en posiciones diferentes de opresión y rol de oprimido simultáneamente ${ }^{25}$. Además, hace referencia a cómo se organizan estas intersecciones en diferentes dominios: estructural, disciplinario, hegemónico e interpersonal, y que si bien dependen del contexto, estos dominios representan el patrón común de la organización de poder y desigualdad en cualquier sociedad.

\section{MATRIZ DE DOMINACIÓN}

Hill-Collins propone cuatro condiciones de matrices de dominación ${ }^{25,26}$ : 
Dominio estructural: Abarca la forma en que las instituciones (escuelas, industrias, hospitales, bancos) se organizan para reproducir la subordinación, por ejemplo de las mujeres negras a través del tiempo, mediante múltiples formas de segregación para reproducir situaciones injustas de exclusión social, como discriminación en el acceso al trabajo, menos paga salarial por las mismas labores que mujeres blancas, prejuicios y determinación en el acceso a unos trabajos sobre otros, etc. Debido a que este dominio es a gran escala, pues abarca todo el sistema y ha operado por largo tiempo a través de instituciones sociales interconectadas, es que el mismo grupo social excluido es quien trata de cambiar las políticas y leyes a través de reformas sociales, en movimientos sociales. Esto es una importante estrategia para la inclusión en el grupo de dominio estructural.

Dominio disciplinario: Este dominio incrementa su importancia gracias a la creciente "oportunidad" que genera la burocracia como modo de organización social moderna, pues ha tomado lugar en el control de las poblaciones, especialmente a través de la raza, el género y otros marcadores de la diferencia. Este medio de organización se vuelve altamente eficaz a medida que reproduce la intersección entre opresiones y enmascara sus efectos. Hill-Collins también añade que la vigilancia a un grupo social constituye un mayor mecanismo de control burocrático, y que la resistencia desde dentro de la organización burocrática forma parte de una estrategia para acabar con la dominación. Ejemplos de ello, son las prácticas sociales que invisibilizan a unos grupos sociales sobre otros, por ejemplo desplazamiento de población negra a zonas periféricas de las grandes ciudades por políticas sociales, invisibilización de la población lesbiana, gay, transexual/ transgénero, bisexual e intersexual (LGTBI) en contextos educativos, etc.

Dominio hegemónico: Este dominio de poder se ocupa de la ideología, la cultura y la conciencia. Apunta a justificar prácticas en estos dominios de poder, al manipular la ideología y la cultura, pues el dominio hegemónico actúa como un vínculo entre las instituciones sociales (dominio estructural), sus prácticas organizativas (dominio disciplinario) y el nivel de interacción social cotidiana (dominio interpersonal) ${ }^{25}$ (p. 284). [Traducción propia]Este dominio argumenta que los grupos dominantes crean y mantienen un sistema popular de ideologías, que sostienen y apoyan el derecho a gobernar de este grupo y la mantención de los sistemas de opresión, a través de currículos escolares, enseñanzas religiosas, culturas comunitarias e historias familiares.

Dominio interpersonal: Plantea en su argumento que aunque la mayoría de los individuos tienen facilidad para reconocer su propia victimización dentro de algún sistema mayor de opresión, no ven cómo sus pensamientos y acciones reproducen la subordinación de otra persona. Hill-Collins refiere que "la dominación actúa seduciendo, presionando o forzando (...) a los miembros de los grupos subordinados y a todos los individuos, a reemplazar las formas individuales y culturales de conocer por el pensamiento del grupo dominante" 25 ( $p$. 287). El dominio interpersonal funciona a través de prácticas rutinarias y cotidianas de relaciones sociales, las cuales son sistemáticas, recurrentes y tan familiares que normalmente pasan desapercibidas. Las estrategias de resistencia dentro de este dominio pueden tomar tantas formas como individuos, pues entra en un nivel cotidiano de acción. 
Es así como el concepto de interseccionalidad revela una carga política que posiciona a las agendas políticas como fuentes de la producción y solución de la desigualdad interseccional. Hay que pensar sobre qué tipo de política dialógica entre los diferentes actores sería necesario para garantizar que la intersección entre desigualdades se incluya en las agendas académicas o de los movimientos.

Esta perspectiva nos permite analizar las experiencias sociales implicadas en las ocupaciones, considerando que existen diversos sistemas opresores que generan desigualdades y condiciones de vulnerabilidad ${ }^{24}$, y que constantemente están presentes en la vida diaria a través de diferentes dominios de poder.

\section{MÉTODO NARRATIVO}

Consideramos que una perspectiva metodológica que permite integrar al transaccionalismo con la interseccionalidad feminista es el método narrativo. Este método, entendido como un proceso de conversación, reflexión y escritura dialógica, permite la reconstrucción de las historias de vida de las personas, dando una importancia crucial a la voz y experiencia de las personas en sus propias historias de vida:

Las personas dan forma a sus vidas cotidianas por medio de relatos sobre quiénes son ellos y los otros, conforme interpretan su pasado en función de esas historias. El relato, en el lenguaje actual, es una puerta de entrada a través de la cual una persona se introduce al mundo y por medio de la cual su experiencia del mundo es interpretada y se transforma en personalmente significativa ${ }^{12}$ (p.139).

Es así, como las narrativas serán el resultado de interpretaciones y reinterpretaciones en forma conjunta entre investigadoras y participantes, dando importancia al carácter situado y dialógico del lenguaje ${ }^{11}$.

A través de las narrativas es posible interpretar las diferentes experiencias de las personas en tanto ejecutan diferentes ocupaciones, junto con sus condiciones de vida, contextos, sistemas de opresión y relación transaccional. Todo esto, desde una perspectiva situada y contextual.

Así, al utilizar el método narrativo la unidad de análisis de la investigación se traduce en la narrativa de las participantes ${ }^{12}$ el que podrá ser interpretado y reconstruido por ellas.

Por otro lado, este método considera el vínculo que se genera entre las investigadoras y las participantes al definirlo como un proceso circular, es decir, a través de la escritura dialógica existe una primera instancia en donde se recoge parte del relato pero éste se le devuelve a la participante para contar con su feedback. 
En este sentido, no se trata de narrar la o las historias de otros/as, sino narrar el diálogo que se ha producido en nuestra propia intervención ${ }^{27}$. De esta forma buscamos una ruptura en la relación jerárquica tradicional entre investigadoras y participantes.

\subsection{Relatos de vida}

Dentro del método narrativo es posible identificar de forma particular el enfoque de los relatos de vida $^{28}$. Este nos permite acceder a la mentalidad de las personas, y al mismo tiempo, nos permite conocer las acciones en sus vidas a través de la descripción de su cotidianidad y relaciones sociales. Además, permite obtener información social, pues es posible estimar detalles de la narrativa, así como eliminar barreras para acceder en el ámbito privado de las personas. Junto con ello, es posible identificar los hechos significativos presentes en el contexto en diferentes situaciones particulares de forma de potenciar la reconstrucción situada de la experiencia, siempre desde una perspectiva interpretativa ${ }^{29}$.

El método narrativo de los relatos de vida se centra en conocer "historias" en la vida de las personas o momentos concretos en su experiencia, incluyendo en el relato la relación con su realidad social, contextos, costumbres, entre otros aspectos ${ }^{30}$. Es así que este método permite profundizar en ciertos momentos relevantes dentro de la vida de las personas, junto con las condiciones que determinaron o influyeron en que estos sucedie$\operatorname{ran}^{29}$. Además, el método hace énfasis en la manera en que la persona da orden a su experiencia a través de su narrativa para otorgarle sentido ${ }^{11}$.

Las narrativas permiten realizar una doble interpretación de momentos biográficos de la persona, siendo el relato de vida la enunciación por parte de la persona participante; y la historia de vida una producción conjunta con las investigadoras, situada en el momento de su producción y que reconstruye la narración en base a categorías ${ }^{27,29}$.

La realización de la historia de vida, y su correspondiente categorización y análisis en base a conceptos, se puede llevar a cabo en base a las constructos que surgen de las perspectivas transaccionalista e interseccional feminista.

En este sentido, esta integración permite revivir y localizarse en la situación relatada, por lo que, se consideran aspectos de lugar, tiempo, y condiciones determinadas para conocer la realidad histórica por donde transita el/la narrador $/ a^{12}$.

Algunos aspectos a considerar en las narrativas es que pueden poner en tensión la relación dicotómica entre "investigadoras y sujetos/as de estudio". Al respecto, es posible poner énfasis en el contexto de producción de las narrativas, la influencia de la/el investigador/a, la circunstancia social de producción e interpretación de las narrativas, cuestionando el "para qué", "cuándo "y "porqué " se están produciendo estas ${ }^{11}$. Además, en el esfuerzo por generar una horizontalidad forzada, la que muchas veces resulta en un aspecto más discursivo que práctico, diferentes autoras ${ }^{11}$ han propuesto que, en vez de omitir esta relación, creando una falsa horizontalidad, se debe visibilizar este entramado de poder para así poder establecer una "negociación democrática" en el proceso de investigación ${ }^{30}$. 
Al mismo tiempo, dentro del proceso de construcción de narrativas, puede generarse una tensión entre la interpelación bidireccional (esperada) y la reflexividad constante. La tensión con esta propuesta se genera en torno a la preocupación feminista de horizontalidad en la relación que permita que la persona participante exprese sus opiniones y preocupaciones sobre el fenómeno o situación y una segunda preocupación feminista de realizar una crítica y mantener una posición política frente a determinadas situaciones. Se plantea que las instancias de producciones narrativas pueden ser un espacio de reflexividad, pero que esto se dificulta cuando no existe una articulación con la persona que de espacio para el debate y la crítica ${ }^{11}$.

La perspectiva transaccional o transaccionalismo, asociado con a las narrativas, nos permiten entender el significado de las ocupaciones de manera más compleja, contextualizada e implicada en la vida de las personas. En ese sentido, "proveé una perspectiva útil desde la cual observa las complejas e interconectadas capas del 'significado" de las ocupaciones $^{31}$ (p.48). Ya que al comprender la indivisibilidad entre personas, ocupaciones y contextos, implícitamente, estamos entendiendo que los significados otorgados a ciertas situaciones incluyen: (1) las propias subjetividades, con quienes nos desempeñamos y las otras personas que confluyen (o han confluido) durante la transacción; (2) las situaciones en las que nos desempeñamos, es decir, considerando el contexto físico, social, virtual, político, económico, etc. donde ocurre la ocupación; y (3) cómo nuestras ocupaciones son contextualizadas y pensadas de manera integral en términos de (a) pasado, contexto socio-histórico, entendiendo cómo se ha comprendido y constituido a lo largo de la historia, integrando la cultura y las tradiciones en ese proceso, (b) presente, qué implica para cada persona al momento de realizarse una ocupación, y (c) futuro, considerando qué expectativas o pretensiones se sugieren frente al desempeño de ocupaciones, esto implica diferentes acciones o proyecciones de objetos ${ }^{31}$.

Además, al integrar la perspectiva interseccional feminista al transaccionalismo, junto con el método narrativo, es posible dejar en evidencia los diferentes sistemas de opresión que operan en el proceso de ejecución de ocupaciones. Es decir, si bien el transaccionalismo y las narrativas nos entregan una mirada histórico-social de las ocupaciones, que permite reconstruir una situación ocupacional que rescata el valor cultural, personal y social de una ocupación, la interseccionalidad nos permite evidenciar cómo estos contextos o diferentes aspectos aparecen en escena determinando los significados o la propia ejecución o imposición en la ejecución de las ocupaciones.

Un ejemplo que integra estas perspectivas puede ser las ocupaciones que desempeñan mujeres migradas en un contexto diferente a su país de origen. Desde esta lógica, tras el uso de narrativas, es posible interpretar cómo el transaccionalismo muestra las ocupaciones desempeñadas desde una cultura y contexto histórico social particular y la interseccionalidad da cuenta de revelar, presentar y promover una lectura de los sistemas desde los relatos de las personas, dando cuenta de los sistemas de opresión que funcionan en la restricción o facilitación en el desempeño ocupacional de las personas. 
Al respecto, una investigación en Chile desarrolló un estudio, a partir de la perspectiva transaccional y metodología narrativa, interesado en las ocupaciones que mujeres migradas desempeñaban en su vida cotidiana eran modificadas de acuerdo al proceso de adaptación cultural y social. Así, la tradición histórica se entrecruza con aspectos contextuales, requerimientos de las situaciones actuales y proyecciones futuras de ocupaciones las que son modificadas, muchas veces, sin la intencionalidad de estas mujeres ${ }^{32}$.

Por otro lado, esto se entrelaza con la perspectiva interseccional que evidenció cómo lo sistemas de opresión Hill-Collins ${ }^{25}$ funcionan limitando las posibilidades ocupacionales de estas mujeres. Las políticas migratorias, como dominios estructurales y disciplinarios expresados en la escasez de apoyos económicos, disminución de facilidades para regularizar legalmente la permanencia en el país, y la compleja burocracia para realizar los trámites de permanencia, junto con los dominios hegemónicos e interpersonales, expresados en prejuicios, racismo, xenofobia y malos tratos en las relaciones de estas mujeres determinan y restringen las ocupaciones que estas mujeres pueden desempeñar. Sumando esto, a una perspectiva de género, se da cuenta cómo los sistemas de opresión poseen trayectorias transaccionalistas que determinan las ocupaciones de estas mujeres.

\section{CONSIDERACIONES FINALES}

El presente artículo presentó como la integración entre las perspectivas transaccionalista, interseccional feminista y método narrativo, en especial los relatos de vida, pueden ofrecer aportes teórico-metodológicos para la investigación en Terapia Ocupacional y en Ciencia Ocupacional, de forma de producir saberes y acciones críticas, coherentes y complejas, con foco en el estudio de las ocupaciones, necesarios para nuestra actualidad global.

Estas perspectivas se revelan coherentes con las miradas críticas que centran el análisis en el lenguaje y que permite estudiar la relación de los procesos históricoculturales en la construcción de identidades y de diferentes experiencias centradas en la ejecución de ocupaciones contextualizadas y considerando su trayecto socio-histórico.

Explorar desde esta perspectiva permite comprender las bases que contribuyen para una sociedad desigual y que dificultan su transformación. Considerando aún que, las narrativas pueden dar voces a las experiencias y los discursos contra hegemónicos y socialmente legitimados.

Todo lo anterior toma especial relevancia para retomar la perspectiva holística fundacional de la Terapia Ocupacional, situando el foco de interés en los diversos elementos entrelazados que producen a la ocupación, más que centrarse solamente en su ejecución o en una individualidad. 
Referencias

1.Morrison R, Olivares D, Vidal D. La filosofía de la Ocupación Humana y el Paradigma Social de la Ocupación. Algunas reflexiones y propuestas sobre epistemologías actuales en Terapia Ocupacional y Ciencias de la Ocupación. The Philosophy of Human Occupation and the Social Paradigm of the Occupation. Some reflections and suggestions on current epistemologies in Occupational Therapy and Occupational Science]. Revista Chilena de Terapia Ocupacional. 2011; 11(2):102-119. DOI:10.5354/07195346.2011 .17785

2.Morrison R. Pragmatist Epistemology and Jane Addams: Fundamental Concepts for the Social Paradigm of Occupational Therapy. Occupational Therapy International. 2016; 23(4):295-304. < DOI: 10.1002/oti.1430>

3.Shank $\mathrm{K}$, Cutchin $\mathrm{M}$. Transactional occupations of older women aging in place: Negotiating change and meaning. Journal of Occupational Science. $2010 ; 17(1): 4-13$. < https://doi.org/10.1080/14427591.2010.9686666>

4.Dickie V. Are occupations 'processes too complicated to explain'? What we can learn by trying. Journal of Occupational Science. 2010; 17(4):195-203. <https:// doi.org/10.1080/14427591.2013.781920>

5.Aldrich R. From complexity theory to transactionalism: Moving occupational science forward in theorizing the complexities of behavior. Journal of Occupational Science. 2008; 15(3):147-156. < https://doi.org/10.1080/14427591.2008.9686624>

6. Kuo A. A Transactional View: Occupation as a Means to Create Experiences that Matter. Journal of Occupational Science. 2018; 18(2):131-138. <https:// doi.org/10.1080/14427591.2011.575759>

7.Esguerra C, Bello JA. Interseccionalidad y políticas públicas LGBTI en Colombia: usos y desplazamientos de una noción crítica. Revista de Estudios Sociales. 2014; 49:19-32.

8. Mora-Ríos J, Bautista N. Estigma estructural, género e interseccionalidad: Implicaciones en la atención a la salud mental. Salud Mental. 2014; 37(4):303-312.

9.Crenshaw K. Mapping the Margins: Intersectionality, Identity Politics, and Violence against Women of Color. Stanford Law Review. 1991; 43(6):1241-1299.

10. McCall L. The Complexity of Intersectionality. Signs: Journal of Women in Culture and Society. 2005; 30(3):1771-1800.

11.Troncoso L, Galaz C, Alvarez C. Las Producciones Narrativas como metodología de investigación feminista en Psicología Social Crítica: tensiones y desafíos. Psicoperspectivas. 2017; 16(2):20-32.

12. Blanco M. Investigación narrativa: una forma de generación de conocimientos. Argumentos (México, DF). 2011; 24:135-156.

13. Kielhofner G. Modelo de ocupación humana: teoría y aplicación [Model of Human Occupation: theory and application]. Buenos Aires: Ed. Médica Panamericana; 2004.

14.Zemke R, Clark F. Occupational science: The evolving discipline. Philadelphia: FA Davis Company; 1996.

15. Dickie V, Cutchin MP, Humphry R. Occupation as transactional experience: A critique of individualism in occupational science. Journal of Occupational Science. 2006; 13(1):83-93. <DOI: 10.1080/14427591.2006.9686573>

16. Cutchin $M$, Dickie $V$. Transactionalism: Occupational science and the pragmatic attitude. In: Whiteford G, Hocking C, eds. Occupational Science: Society, Inclusion, Participation. Oxford: Wiley-Blackwell; 2012. <https://www.tandfonline.com/doi/ abs/10.1080/14427591.2006.9686573> 
17.Galheigo S. Perspectiva critica y compleja de Terapia Ocupacional, actividad, cotidiano, diversidad, justicia social y compromiso etico y politico. Revista Terapia Ocupacional Galicia. 2012; Monográfico 5:176-187. <http://www.revistatog.com/mono/ num5/compromiso.pdf>

18.Guajardo A, Galheigo SM. Reflexiones críticas acerca de los derechos humanos: Contribuciones desde la terapia ocupacional Latinoamericana [Critical Reflections on Human Rights: Contributions from Latin American Occupational Therapy]. World Federation of Occupational Therapists Bulletin. 2015; 71(2):73-80.

19. Morrison R, Guajardo A, Schliebener M. Conferencia: Debates y reflexiones para una Ciencia de la Ocupación crítica y social. Diálogos para comprender la Ocupación Humana [Conference Proceedings: Debate and reflections for a critical and social Occupational Science. Dialogues to understand Human Occupation]. Revista Argentina de Terapia Ocupacional [Argentine Journal of Occupational Therapy]. 2016; 1(2):40-58.

20.Galvaan R. Occupational choice: the significance of socio-economic and political factors. In: Whiteford G, Hocking C, eds. Occupational Science: Society, Inclusion, Participation. Oxford, UK: Wiley-Blackwell; 2012: 152-161.

21. Morrison R. Terapia Ocupacional y Pragmatismo. Contribuciones teóricas para la práctica. Santiago de Chile. Editorial Universitaria; 2017.

22. Heidegger M. Ser y tiempo. $4^{\mathrm{a}}$. ed. Santiago. Universitaria; 2005.

23.Dewey J. The school and society. En Ann Boydston (Ed.) (2008), Middle works of John Dewey 1899-1924. $1^{\text {a }}$ ed. Carbondal. Southern Illinois Universiry Press; 1899.

24. Magliano MJ. Interseccionalidad y migraciones: potencialidades y desafíos. Revista Estudos Feministas. 2015; 23:691-712.

25. Hill-Collins P. Black Feminist Thought: Knowledge, Consciousness and the Politics of Empowerment. New York: Routledge; 1990.

26. Hill-Collins P. Gender, black feminism, and black political economy. The Annals of the American Academy of Political and Social Science. 2000; 568(1):41-53.

27. Haraway D. Saberes localizados: a questão da ciência para o feminismo e o privilégio da perspectiva parcial. Cadernos pagu. 1995; (5):7-41.

28. Cornejo M. El enfoque biográfico: trayectorias, desarrollos teóricos y perspectivas. Psykhe (Santiago). 2006; 15(1):95-106.

29. Cornejo M, Mendoza F, Rojas RC. La investigación con relatos de vida: pistas y opciones del diseño metodológico. Psykhe (Santiago). 2008; 17(1):29-39.

30.Cordero MC. Historias de vida: Una metodología de investigación cualitativa. Revista Griot (Etapa IV-Colección completa). 2012; 5(1):50-67.

31. Hocking C, Reed K. Resituating the Meaning of Occupation: A Transactional Perspective. In: Cutchin M, Dickie V, eds. Transactional Perspectives on Occupation. New York: Springer; 2013: 39-50.

\section{Herrera A, Llambias V, Morrison R. Mujeres migradas en Chile: experiencias y} ocupaciones cotidianas desde la interseccional feminista [Tesis de grado]. Santiago de Chile: Departamento de Terapia Ocupacional y Ciencia de la Ocupación, Universidad de Chile; 2017. 
* Este documento es parte del marco teórico una investigación mayor, llamada: Mujeres migradas en Chile: experiencias y ocupaciones cotidianas desde la interseccional feminista. En el marco de la Tesis de grado de Almendra Herrera Ferrufino y Viviana Llambias Miranda, dirigida por Rodolfo Morrison y financiada por el Departamento de Terapia Ocupacional y Ciencia de la Ocupación de la Universidad de Chile (2017).

Contribución de las autoras y autor: Almendra Ferrufino, Viviana Miranda, Rodolfo Morrison y Gabriela Yates, realizaron la búsqueda, la selección y el análisis de la información. Rodolfo Morrison estructuró y editó el documento. Carla Silva realizó aportes teóricos al documento, realizo su revisión y edición final y lo tradujo al portugués. Todas las autoras aprobaron su versión final.

Sometido en: 17/04/2018

Aceptado en: 09/08/2018

Publicado en: $31 / 01 / 2019$ 